\title{
Itinerarios terapéuticos de madres qom en una comunidad periurbana de Formosa
}

Therapeutic itineraries of Qom mothers in a peri-urban community of Formosa

\author{
Estud. Shalila de Bourmont ${ }^{a}$, Lic. Sofía Olmedo ${ }^{b}, M g$. Penélope Rodríguez ${ }^{c}$ y \\ Dra. Claudia Valeggia ${ }^{d}$
}

\section{RESUMEN}

Introducción. Las poblaciones indígenas están atravesando profundos cambios en su estilo de vida que afectan la salud y la manera en que tratan sus enfermedades. El objetivo del estudio fue determinar los itinerarios terapéuticos que las madres qom siguen cuando perciben que sus hijos están enfermos.

a. Facultad de Medicina, Universidad de California, San Francisco, CA EE. UU.

b. Centro de Investigaciones y Estudios sobre Cultura y Sociedad, Universidad Nacional de Córdoba, Argentina.

c. Hilo Counseling Center, Hilo, Hawaii, EE. UU.

d. Department of Anthropology, Yale University, New Haven, Connecticut, EE. UU.

Correspondencia: Dra. Claudia Valeggia: claudia.valeggia@yale. edu

\section{Financiamiento:}

Este estudio fue parcialmente financiado por la Universidad de Yale, a través de una beca para estudios independientes otorgada a SDB y por un subsidio de la National Science Foundation otorgado a CV (NSF BCS-0952264).

Conflicto de intereses: Ninguno que declarar.

Recibido: 3-8-2019

Aceptado: 26-12-2019
Cómo citar: de Bourmont S, Olmedo S, Rodríguez P, Valeggia C. Itinerarios terapéuticos de madres qom en una comunidad periurbana de Formosa. Arch Argent Pediatr 2020;118(4):240-244. http: / / dx.doi.org/10.5546/ aap.2020.240

http:/ / dx.doi.org/10.5546/ aap.2020.eng.240$$
\text { . }
$$

\section{INTRODUCCIÓN}

A $n$ i v e $1 \quad m$ u d i a 1 , independientemente de su condición de vida, casi 400 millones de pueblos originarios tienen estándares de salud muy bajos. ${ }^{1,2}$ Estas inequidades sanitarias afectan, en particular, a las mujeres y a los niños indígenas, quienes sufren mayores tasas de mortalidad infantil, de desnutrición y de mortalidad materna que sus pares no indígenas. ${ }^{3}$

Las comunidades qom de nuestro país no son la excepción. Como ciudadanos argentinos, tienen acceso a servicios médicos gratuitos en hospitales públicos y centros de salud. ${ }^{4}$ La mayoría de los qom viven en las provincias de Chaco y Formosa, las cuales presentan los peores indicadores de salud del país. ${ }^{5} \mathrm{~A}$ medida que avanzan hacia un estilo de vida más globalizado e integrado en el mercado occidental, las madres originarias se enfrentan a una variedad de sistemas de salud que incluyen tanto modelos tradicionales como occidentales.

Una mejor comprensión de la compleja interacción entre las variables culturales, sociales y estructurales que inciden en esas decisiones puede contribuir a optimizar la coordinación a través de múltiples enfoques y disminuir la carga de las inequidades en temas de salud que afectan a estos pueblos originarios. En este estudio, el objetivo fue determinar los factores que influyen en las elecciones de las madres qom respecto a la atención médica para sus hijos/as. 


\section{MÉTODOS}

Población de estudio: Este estudio se realizó en Namqom, un asentamiento qom de 3500 personas, ubicado a $11 \mathrm{~km}$ al oeste de la ciudad de Formosa, al norte de la Argentina. Aunque hablaban su lengua materna como primer idioma, la gran mayoría también hablaba español de manera fluida. Todas las familias en Namqom estaban por debajo del umbral de pobreza en la Argentina y sufrían discriminación e injusticia social. ${ }^{6,7}$ Dentro del barrio, los residentes podían acceder fácilmente a la atención biomédica occidental en el centro de salud y en los hospitales de la ciudad, los que dependían del Ministerio de Desarrollo Humano de la provincia. Los programas de atención prenatal y posnatal eran gratuitos y se llevaban a cabo en el centro de salud barrial y en el hospital de la ciudad, ubicado a unos $15 \mathrm{~km}$ de Namqom.

Diseño del estudio: Se trató de un estudio exploratorio cualitativo, basado en un modelo de creencias de salud. ${ }^{8}$ Se utilizó una estrategia de muestreo intencional para seleccionar a las madres. Los participantes se seleccionaron de una cohorte existente de 150 madres involucradas en un proyecto de investigación en curso llevado a cabo por el Programa de Ecología Reproductiva de Chaco Argentino. De esta cohorte, se creó un subconjunto eligiendo a todas las madres con niños menores de 5 años. El tamaño de la muestra se seleccionó sobre la base de la saturación teórica. ${ }^{9}$

Recopilación de datos: Las entrevistas se llevaron a cabo en el hogar de las madres durante los meses de mayo a agosto de 2017. Se utilizó una entrevista semiestructurada abierta y anónima (véase el Anexo en formato electrónico).

Consideraciones éticas: Se realizó una declaración de consentimiento por escrito a todos los participantes antes de la entrevista y también se solicitó el consentimiento verbal. El protocolo fue aprobado por el Comité de Ética de la Universidad de Yale bajo el número 1501015214.

Análisis: Teniendo en cuenta la metodología analítica de estudios previos sobre salud y cultura, se utilizó un marco conceptual a partir de una lista de códigos predeterminados. ${ }^{10-12}$ Sucesivamente, se complementó con el uso de la teoría fundamentada (grounded theory) a partir del contenido de la entrevista a medida que surgieron nuevos patrones y temas. A lo largo de este proceso, se utilizó el método comparativo constante para asegurar que la codificación permaneciera constante y que la estructura del código evolucionara inductivamente. La codificación se llevó a cabo utilizando el programa NVivo.

\section{RESULTADOS}

Un total de 16 madres qom constituyeron nuestra muestra. La edad promedio de las entrevistadas fue de 32,8 años (rango: 23-45 años) y sus hijos más pequeños tenían entre 3 meses y 4 años de edad. La paridad promedio fue de 4 niños/as (rango: 1-11). Catorce madres tenían cierta escolaridad formal, aunque solo 5 completaron la escuela secundaria. La mayoría de las participantes estaban casadas o en pareja.

\section{Factores que influían en la elección de la acción terapéutica}

Se identificaron dos factores que influían en la elección de la madre:

a) Percepción de signos de dolencia y determinación de la gravedad de los sintomas

Las madres mencionaron signos físicos y emocionales como indicadores de enfermedad de sus hijos/as. Por ejemplo, referían la apariencia de los ojos como un claro indicador de enfermedad y los describían como "aburridos" o "vidriosos" cuando el niño no estaba bien de salud. Si bien la percepción de las señales físicas y emocionales de la enfermedad era suficiente en algunas madres para comenzar la búsqueda de atención médica, la presencia de síntomas particulares parecía ser necesaria para comenzar a involucrarse. La fiebre, la diarrea y el vómito se mencionaron como los síntomas más preocupantes, ya que se creía que causaban letargo, debilidad y pérdida de peso.

b) Comprensión de la etiología de las enfermedades

Según la información proporcionada, las enfermedades se clasificaron como naturales o no naturales. Las causas naturales se caracterizaron como de causas externas y directamente vinculadas con el medioambiente (frío, viento, humedad). Estas causas se asemejaban a la comprensión biomédica de la etiología de la enfermedad. Las enfermedades de origen no natural se referían a una interpretación más folklórica o indígena tradicional de las enfermedades. Algunos ejemplos: a) "Vuelta del vasito de leche", caracterizado por vómito, diarrea y fiebre. Esta enfermedad ocurría cuando un supuesto vaso de leche dentro del estómago del niño se volcaba debido a movimientos bruscos del niño. b) "Ojeo" o "mal de ojo", que ocurría cuando una persona malvada, un hombre ebrio 
o una mujer que estaba menstruando miraban a alguien. Se desarrollaba durante el embarazo o durante la primera infancia. Según las madres, los médicos occidentales solían diagnosticar "mal de ojo" como meningitis. c) "Empacho", que se caracterizaba por indigestión, estreñimiento o diarrea; a menudo, se atribuía al consumo de alimentos "pesados" (grasos) y se percibía como un bolo alimenticio no digerido que estaba atorado dentro del tracto digestivo. El "cuarurú" se manifiestaba a través de síntomas similares. Se creía que era causado por el consumo excesivo de pollo durante el embarazo, pero solo se manifestaba después del nacimiento durante la primera infancia.

\section{Relación entre prevención y etiología de la enfermedad}

La prevención estuvo casi totalmente ausente dentro del concepto de salud de las madres. La lactancia materna, que se practicaba ampliamente en la comunidad, no se mencionó como una medida preventiva. Además, la noción de que la enfermedad era inevitable fue prevalente en las entrevistas. Los chequeos o controles en el centro de salud tampoco se consideraban una forma de prevención de enfermedades. De hecho, algunas madres informaron que no llevaban a sus hijos a la sala para el chequeo mensual porque "estaban sanos". La buena nutrición, la higiene y las condiciones de sanidad, rara vez, se mencionaron como estrategias de prevención.

\section{Selección del sistema de salud}

Los sistemas de curación que se mencionaron como un primer curso de acción en orden de frecuencia de respuestas fueron los siguientes:

1. Centro de salud: Era el primer curso de acción para la mayoría de las madres. A pesar de la falta de consenso sobre la calidad y la eficacia del servicio, las madres elegían este sistema por la familiaridad con el servicio, la accesibilidad, la disponibilidad y el acceso a recursos adicionales. Las críticas al centro de salud más citadas por las madres incluyeron las siguientes: a) Falta de atención y respeto del personal médico, que incluía las largas horas de espera para ser atendidas; $b$ ) Inconsistencias en el servicio (no había médicos de guardia durante la noche ni durante los fines de semana o feriados; la farmacia no estaba bien abastecida y faltaban medicamentos); c) Incapacidad para curar enfermedades tradicionales: las madres mencionaron sentirse frustradas cuando los biomédicos no reconocían las enfermedades tradicionales, como la del "vasito de leche".

2. El hospital: Las opiniones sobre el hospital fueron, en su mayoría, favorables e incluían alta calificación y atención del personal, disponibilidad y accesibilidad de los recursos, familiaridad y comodidad con los servicios. Para ir al hospital de la ciudad, las madres viajaban en la moto de su familia o tomaban un colectivo público.

3. Tratamientos en el hogar: Se encontró que los medicamentos farmacéuticos se usaban como primera instancia, seguidos por remedios caseros y plantas medicinales. Los medicamentos se compraban sin receta y, generalmente, se creía que eran más efectivos y apetecibles para un niño que los que administraban en el centro de salud. La mayoría de los medicamentos que conseguían eran de origen paraguayo; entre los más frecuentes, se encontraban el paracetamol (Medifar), Z-Cal ${ }^{\circledR}$ (Indufar), la Lascamicetina ${ }^{\circledR}$ (Lasca) y Mentolina ${ }^{\circledR}$ ungüento (Catedral). En las farmacias, compraban Aspirinetas, ibuprofeno, QuraPLus ${ }^{\circledR}$, Pulmosan ${ }^{\circledR}$ y jarabes varios. Los remedios caseros se utilizaban después de llevar a los niños al centro de salud y como una forma de complementar el tratamiento biomédico. Las plantas medicinales se usaban, principalmente, para problemas gastrointestinales, como dolores de estómago, diarrea, vómitos y parásitos. Los datos mostraban que las madres dudaban ante este tipo de remedios naturales, debido a los antecedentes de intoxicación por el uso de pantas medicinales.

4. Curanderos (sanadores tradicionales): Los relatos de las madres sobre el tratamiento del "vasito de leche" mostraron una importante consistencia. El tratamiento por parte de los curanderos consistió en una realineación especializada de las piernas de un niño en combinación con medicamentos a base de hierbas y el uso de una faja, y la duración del tratamiento era de tres días.

Los "curanderos" eran percibidos como figuras de la autoridad de salud que eran respetadas. La conexión entre el curandero y el enfermo era tan fuerte que se creía que la enfermedad podía transferirse. Las madres usaban "curanderos", principalmente, para tratar el "vasito de leche". En qom, la persona que podía curar (y también causar) hechizos y brujerías se denominaba pi'ogonaq. Los pi'ogonaq eran particularmente temidos, pero, rara vez, estaban involucrados con enfermedades infantiles. 
5. Curación religiosa: La curación religiosa, que consistía en sesiones de oración en la iglesia (evangélica o pentecostal) donde se leían pasajes de la biblia, la practicaban personas que invocaban al Espíritu Santo y estaban dotadas de su poder. Los tipos de curación religiosa más citados por las madres incluían reanimación, visiones y bautismo. Las madres relataban que las enfermedades se podían prevenir por medios religiosos.

\section{Interacciones entre sistemas}

Las madres contaban que no había conflictos entre el uso del sistema biomédico, la curación religiosa y el "curandero", ya sea de manera simultánea o consecutiva. De hecho, las madres, a menudo, informaron que los "curanderos" les indicaban asistir al centro de salud después del tratamiento para tratar cualquier fiebre residual que se creía que era el resultado del tratamiento tradicional intensivo.

Sin embargo, la curación religiosa (bíblica) y la curación tradicional (chamánica) se consideraban inherentemente en desacuerdo, debido al hecho de que las madres veían a los curanderos como agentes curativos dotados del poder de espíritus malévolos o satánicos. Además, las interacciones entre los dos sistemas podían resultar en un daño físico al "curandero" tradicional. La excepción a esta relación tenía que ver con el uso de un "curandero" para "el vasito de leche", debido a su etiología.

\section{Eficacia percibida de los sistemas de salud}

La relación entre la capacidad percibida de un sistema de salud y la selección del sistema no era directa. La mayoría de las personas elegían el sistema biomédico de salud cuando los niños estaban gravemente enfermos y los síntomas empeoraban. Solo después, cuando una enfermedad no se curaba por la medicina occidental, se consideraba que se debía a causas sobrenaturales y se consultaba a un curandero tradicional o religioso. En cambio, cuando se trataba del sistema religioso, la fe parecía jugar un papel importante y la mayoría de las madres confiaban inequívocamente en los "curanderos" religiosos.

Respecto a los "curanderos tradicionales", todas las madres entrevistadas tuvieron experiencias con estos sanadores y afirmaron que su tratamiento siempre había funcionado. Además, cuando describieron explícitamente los tratamientos tradicionales, parecían aceptarlos por completo sin cuestionarlos. Sin embargo, la aceptación casi universal de la efectividad de un "curandero" no se traducía en una utilización universal de sus servicios. De hecho, debido a que se creía que los "curanderos" tenían la capacidad de sanar y dañar, el temor a ellos en algunas madres se traducía en una desconexión con sus servicios. Por lo tanto, las entrevistas sugirieron que tanto el miedo como la confianza en la efectividad jugaban un papel importante en la selección de una estrategia de salud.

\section{DISCUSIÓN}

Este estudio representó una etapa inicial para la indagación respecto al pluralismo médico presente en estas comunidades originarias. Permitió identificar los factores principales que influían en la determinación de los itinerarios terapéuticos para seguir por las madres qom. Se encontró que tanto la búsqueda de tratamiento como la práctica y adherencia a este se relacionaban no solo con la historia natural de la enfermedad, sino también con aspectos socioculturales, ${ }^{13}$ que, muchas veces, eran descuidados o directamente rechazados por el sistema médico hegemónico.

La visita al centro de salud y la automedicación constituían el primer curso de acción de las madres qom, debido a la comprensión de la enfermedad desde la etiología natural. Este resultado, que concordó con un estudio previo, ${ }^{14}$ mostró cierta receptividad hacia el enfoque biomédico, ${ }^{15}$ aunque la comunidad no tuviera una total aceptación. Sin embargo, si la medicina biomédica no otorgaba una mejoría de los síntomas rápidamente, el origen de la enfermedad pasaba a ser interpretado como no natural y se cambiaba el rumbo hacia la curación tradicional. En este estudio, se evidenció una consistencia en el uso del curandero para el tratamiento del "vasito de leche" o el "empacho", como sucedió en otras poblaciones de la Argentina. ${ }^{16-18}$

El conocimiento de las madres qom respecto a la etiología occidental de las enfermedades era limitado, con una marcada ausencia de la noción de prevención. En este punto, fue importante tener en cuenta que las comunidades originarias tenían una visión integral de la salud, en la que el aspecto espiritual ocupaba un lugar determinante. ${ }^{19}$

En este estudio, al igual que en un estudio previo, ${ }^{14}$ se evidenció que los itinerarios terapéuticos en un sistema de pluralismo médico no eran necesariamente incompatibles. Las 
personas recurrían a diferentes itinerarios no solo para diferentes problemas, sino para el mismo problema de salud..$^{20}$ Este concepto de "triangulación" representaría un potencial punto de encuentro entre la medicina occidental y la comunidad.

Los hallazgos de este estudio brindaron información preliminar sobre las interacciones complejas que podían ocurrir en un sistema de salud pluralista. Una limitación del estudio se debió al marco de tiempo del proyecto, así como a las limitaciones inherentes a un estudio cualitativo. Una comprensión más profunda de este problema puede ayudar a un mejor diálogo entre el sistema biomédico y la comunidad, $y$, de esa forma, recorrer de manera conjunta (como pares) el camino de la salud.

\section{REFERENCIAS}

1. Stephens C, Porter J, Nettleton C, Willis R. Disappearing, displaced, and undervalued: a call to action for Indigenous health worldwide. Lancet. 2006; 367(9527):2019-28.

2. Valeggia C, Snodgrass JJ. Health of Indigenous Peoples. Annu Rev Anthropol. 2015; 44:117-35.

3. Inter-agency support group on Indigenous Peoples' issues. The Health of Indigenous peoples. In Thematic Paper towars the preparation of the 2014 World Conference on Indigenous Peoples. 2014;1-14. [Acceso: 27 de diciembre de 2019]. Disponible en: https://www.un.org/en/ga/69/ meetings / indigenous / pdf / IASG\% 20Thematic $\% 20$ Paper\%20-\%20Health\%20-\%20rev1.pdf.

4. Valeggia C, Ellison PT. Lactational amenorrhoea in wellnourished Toba women of Formosa, Argentina. J Biosoc Sci. 2004; 36(5):573-95.

5. Ministerio de Salud. OPS.OMS. Indicadores Básicos. Argentina 2017. Buenos Aires. 2017;1-11. [Acceso: 27 de diciembre de 2019]. Disponible en: http:// www.deis.msal.gov.ar/wp-content/uploads / 2018/04/ IndicadoresBasicos2017.pdf.

6. Delucchi M, Fontan M, Grichener S, Wassner M. Proyecto deSaneamiento Básico Integral: Barrio Namqom, Formosa. Formosa, Argentina: Convenio SDS-UNICEF, 1996.

7. Alarcón-Muñoz AM, Vidal-Herrera AC. Dimensiones culturales en el proceso de atención primaria infantil: perspectivas de las madres. Salud Pública Méx. 2005; 47(6):440-6.

8. Rosenstock IM. Why people use health services. Milbank Mem Fund Q. 1966; 44(3):Suppl:94-127.

9. RussellHB. Research Methodsin Anthropology: Qualitative and Quantitative Approaches. 4th ed. Lanham: Altamira Press; 2006.

10. Dell'Arciprete A, Braunstein J, Touris C, Dinardi G, et al. Cultural barriers to effective communication between Indigenous communities and health care providers in Northern Argentina: an anthropological contribution to Chagas disease prevention and control. Int J Equity Health. 2014; 13:6.

11. Bonilla-Castro E, Rodriguez Sehk P. Más Allá del Dilema de los Métodos: La Investigación en Ciencias Sociales. $3^{\text {ra }}$ ed. Bogotá D.C:Universidad de los Andes, Grupo Editorial Norma; 2005.

12. Pope C, Ziebland S, Mays N. Qualitative research in health care. Analysing qualitative data. BMJ. 2000; 320(7227): 114-6.

13. Lago LM, Martins J, Schneider DG, Carvalho Barra DC, et al. Intinerario terapeutico dos usuarios de una urgencia hospitalar. Cienc Saude Coletiva. 2010; 15(Supl 1):1283-91.

14. Olmedo S, Berra S, Valeggia C. Perspectivas de madres Qom sobre la salud infantil. En: Abbona A, Roca I (eds.). Los pueblos indígenas de Ameria Latina Actas del CIPIAL. Santa Rosa: EdUNLPam; 2018.Págs.1-29.

15. Menéndez EL. Modelo médico hegemónico y atención primaria. Segundas Jornadas de Atención Primaria de la Salud. 30 de abril al 7 de mayo de 1988. Buenos Aires; 1988. Págs.451-64.

16. Martinez GJ. Pluralismo médico y etnomedicina entre los Tobas (Qom) del río Bermejito (Chaco, Argentina). Desafíos y aportes para una gestión intercultural de la salud en el impenetrable chaqueño. Rev Mus Antopol. 2011; 4(1):195-210.

17. Arteaga F. Las medicinas tradicionales en la Pampa Argentina. Reflexiones sobre síntesis de praxis y conocimientos médicos, saberes populares y rituales católicos. AIBR. 2010; 5(3):397-429.

18. Idoyaga Molina A. Etiologías, síntomas y eficacia terapéutica. El proceso diagnóstico de la enfermedad en el Noroeste Argentino y Cuyo. Mitológicas. 2001; 16(1):9-43.

19. Stivanello MB. Aportes al debate de la Interculturalidad en salud. Margen. 2015; 76:1-8.

20. Menéndez EL. Modelos de atención de los padecimientos: de exclusiones teóricas y articulaciones prácticas. Cienc Saúde Colectiva. 2003; 9(1)185-207. 


\section{ANEXO \\ Guía de entrevista}

Las entrevistas fueron semiestructuradas. Las conversaciones fueron dirigidas para asegurar que los participantes hablaran sobre los temas delineados abajo.

\section{Introducción:}

Buenos días/tardes. Mi nombre es xxx. Estamos haciendo un estudio sobre la salud de los niños en la comunidad y lo que hacen las mamás cuando sus hijos se enferman. Le agradecería mucho si pudiera contestarme algunas preguntas. Sus respuestas serán de mucha ayuda para entender mejor las necesidades de las madres cuando tienen que atender a un hijo enfermo. Todo lo que nos cuente es anónimo, o sea que su nombre no aparecerá en los resultados del estudio.

\section{Sección demográfica}

1. ¿Cuántos años tiene?

2. ¿Cuántos hijos tiene y de qué edades?

3. ¿Fue usted al colegio? ¿Hasta qué grado/año estudió?

4. ¿Con quién vive en la casa? (Para definir tamaño y composición de la familia).

5. ¿Es usted casada/unida, soltera o viuda?

\section{Nociones generales de salud y enfermedad}

1. ¿Qué significa para usted que una persona tenga "buena salud"?

2. ¿Qué significa para usted estar enfermo?

3. ¿Qué tan importante es para usted tener buena salud?

4. ¿Qué hace usted para que su niño/a se mantenga sano/a?

5. He oído que algunas personas hablan de "enfermedades de blancos". ¿Ha oído usted hablar de esto? ¿Qué significa que una enfermedad sea "blanca"?

\section{Creencias y conocimientos sobre enfermedades infantiles en la comunidad}

1. ¿Qué tanto cree usted que llevar a su hijo al médico (al curandero) lo ayuda a no enfermarse?

2. Para los niños/as, ¿cuáles son las enfermedades que más les dan?

3. ¿Por qué los niños se enferman de ......?

\section{Percepciones sobre la gravedad de los síntomas presentados por los niños}

1. ¿Cuándo comienza usted a preocuparse o a pensar que lo que tiene su niño/a es serio o grave? ¿Por qué?

El curso de acción tomado al evaluar la seriedad de síntomas

1. ¿Qué es lo primero que hace cuando alguno de sus hijos / as se enferma?

2. ¿Con quién consulta inmediatamente cuando alguno de sus hijos / as se enferma: familiares o amigos?

3. ¿Cuándo utiliza usted los remedios caseros? ¿Qué remedios caseros usa? ¿Para qué enfermedades?

4. ¿Cuándo fue la última vez que alguno de sus hijos/as se enfermó de algo serio?

- Cuénteme qué hizo.

- ¿Qué la hizo tomar esa decisión? ¿Alguien la ayudó a tomar esa decisión?

- Y, luego, ¿qué pasó?

\section{Experiencias previas con los diferentes sistemas de salud}

Si la madre buscó ayuda, ya sea en el centro de salud o a un curandero, se preguntó lo siguiente:

- ¿Cómo fue su experiencia en esa ocasión?

- ¿Qué le dijo el curandero (médico) y que le recomendó?

- ¿Qué tanto le sirvieron los remedios al niño? Si los remedios no le sirvieron al niño, ¿qué hizo después?

- ¿Cómo se sintió atendida? ¿Qué le gustó y no le gustó de la experiencia? 
Dependiendo de dónde la madre llevó su niño/a, se preguntó sobre su experiencia con el otro sistema de salud (ej.: si la madre llevó a su niño/a al centro de salud, se preguntó sobre su última experiencia con el curandero).

- ¿Por qué llevó a su hijo al curandero (médico) en esta ocasión?

- Cuénteme más sobre esta experiencia.

- ¿Qué le dijo el curandero (médico) y qué le recomendó?

- ¿Qué tanto le sirvieron los remedios al niño/a? Si los remedios no le sirvieron al niño/a, ¿qué hizo después?

- ¿Cómo se sintió atendida? ¿Qué le gustó y no le gustó de la experiencia? 